\title{
Valorisation des produits locaux dans la lutte contre la malnutrition aigüe modérée dans le Région de Koulikoro
}

\section{Promotion of local products in the fight against moderate acute malnutrition in the Koulikoro Region}

\author{
Traoré SS ${ }^{1}$, Sangho $\mathrm{O}^{2,3}$, Sangho F ${ }^{2,3}$, Ag Iknane $\mathrm{A}^{2,3}$, Coulibaly $\mathrm{D}^{2}$, Coulibaly $\mathrm{CA}^{2}$, Sangho $\mathrm{A}^{3}$, Diallo $\mathrm{B}^{4}$, Coulibaly $\mathrm{H}^{5}$, Coulibaly $\mathrm{M}^{6}$
}

1. Master Santé Publique, Nutrition, DERSP, FMOS, USTTB, Bamako, Mali

2. Département d'Enseignement et de Recherche en Santé Publique, FMOS, USTTB, Bamako, Mali

3. Faculté de Pharmacie, USTTB, Bamako, Mali

4. Centre Hospitalo-Universitaire Odonto-Stomatologie (CHUCNOS), Bamako, Mali

5. Centre de Santé de Référence (CSRéf) de Koulikoro épidémiologiste de terrain niveau intermédiaire, Mali

6. Chargé de cours de Nutrition, DERSP, FMOS, Bamako, Mali

Auteur correspondant : Dr Stanislav Seydou Traoré traorestanis@gmail.com

\section{Résumé}

Introduction: L'utilisation des produits locaux est fortement préconisée dans la lutte contre la malnutrition. Le but de cette étude était de contribuer à la valorisation des produits locaux dans la lutte contre la malnutrition aigüe modérée dans la région de Koulikoro. Matériel et Méthodes : Cette étude s'est déroulée du 1er Novembre 2017 au 31 Mars 2018 dans un village du cercle de Koulikoro. Une étude a été conduite auprès de 12 enfants atteints de malnutrition modère vivants dans les villages à proximité du Centre de Sante Communautaire (CSCOM) de Tienfala. Elle était basée sur un test de dégustation et d'appréciation de trois formules de bouillies par ces enfants. Ces formules étaient à base de niébé, maïs, sorgho et de soja. Cette dégustation devrait permettre d'identifier la formule la plus appréciée qui pourrait être utilisée comme aliment pour lutter contre la malnutrition des enfants. Résultats: La prévalence moyenne de la malnutrition aigüe globale était de 1,3\%. Les cercles les plus touchés étaient Dioïla, Kolokani, Banamba et Nara avec des prévalences moyennes respectives de 2,$2 ; 1,9 ; 1,8$ et $1,1 \%$. Trois formules alimentaires de bouillies à base de farines enrichies de produits locaux ont été utilisées. Leurs valeurs énergétiques étaient de 1055,7 kcal ; 1063,9 kcal et 1013,1 kcal. Elles ont été acceptées et appréciées par les enfants lors de la séance de dégustation avec une préférence pour la première formule. Conclusion : La présente étude a consisté à déterminer l'état nutritionnel des enfants de 6-59 mois et proposer des formules d'aliments de haute valeur nutritionnelle à base des produits locaux.

Mots clés : malnutrition aiguë, MAM, communautaire, Koulikoro.

\section{Abstract:}

Introduction: The use of local products is strongly recommended to fight against malnutrition. This study aimed to contribute to the promotion of local products in the fight against moderate acute malnutrition in the Koulikoro Region. Material and methods: This study took place from November 1, 2017 to March 31, 2018 in a village in the Koulikoro circle. A study was carried out on 12 children suffering from moderate malnutrition living in villages near the Community Health Center (CSCOM) of Tienfala. It was based on a tasting and appreciation test of three porridge formulas by these children. These formulas were based on cowpea, corn, sorghum and soybeans. This tasting should identify the most popular formula that could be used as a food to fight malnutrition in children. Results: The average prevalence of overall acute malnutrition was $1.3 \%$. The most affected circles were Dioila, Kolokani, Banamba and Nara with a respective average prevalence of $2.2 ; 1.9 ; 1.8$ and $1.1 \%$. Three formulas of porridge based on flour enriched with local products were used. Their energy values were $1055.7 \mathrm{kcal} ; 1063.9 \mathrm{kcal}$ and $1013.1 \mathrm{kcal}$. They were accepted and appreciated by the children during the tasting session with a preference for the first formula. Conclusion: The present study consisted of determining the nutritional status of children aged 6-59 months and proposing food formulas of high nutritional value based on local products.

Key words: Acute malnutrition, MAM, communautary, Koulikoro

\section{Introduction :}

En 2016, 156 millions d'enfant de moins de 5 ans souffraient de retard de croissance dans le monde (1). Les enfants avec une malnutrition aiguë modérée (MAM) avaient trois fois plus de risque de mourir des conséquences que ceux bien nourris (1). Ce risque s'élevait à 11,6 fois en cas de malnutrition aiguë sévère (MAS) (1).

Dans leur déclaration commune, les organismes du système des nations unies trouvent qu'il est possible de faire baisser la prévalence de la malnutrition jusqu'à $5 \%$ à travers l'implication de la communauté dans sa gestion (2). L'utilisation des produits locaux est fortement préconisée dans la lutte contre la malnutrition (3). La faisabilité de l'implication de la communauté dans la gestion de la malnutrition au Bangladesh a été étudiée (4). Elle a abouti à des recommandations de sa mise à échelle (4). L'expérience de prise en charge communautaire de la malnutrition en Afrique SubSaharienne a été concluante avec l'amélioration des taux de guérison et de la réduction des taux de décès au Ghana, Malawi, et Zambie en 2014 (5).

Au Mali comme dans la plupart des pays de l'Afrique subsaharienne, la malnutrition constitue un problème de 
santé publique (6). Elle est une des causes majeures de morbidité et de mortalité chez les enfants de moins de cinq ans. II s'agit d'un problème de santé à dimension multifactorielle dont les causes sous-jacentes sont familiales et communautaires dont le manque d'accès à une alimentation de qualité, les soins et pratiques inappropriés d'alimentation du nourrisson et du jeune enfant, les mauvaises pratiques d'hygiène et d'assainissement, l'insuffisance d'accès à l'eau potable et aux services de santé (6). L'enquête Démographique et de Santé du Mali en 2013 (EDSM V) (6) a rapporté sur le plan national une prévalence de $12,7 \%$ de Malnutrition Aigüe Globale (MAG) avec 5,1\% de Malnutrition Aigüe Sévère (MAS) et dans la région de Koulikoro une prévalence de $13,4 \%$ de MAG avec $4,5 \%$ de MAS. Les enquêtes nutritionnelles et de mortalités rétrospectives (SMART) ont indiqué sur le plan national, une prévalence de $12,4 \%$ de MAG avec $2,8 \%$ de MAS en 2015 contre $10,7 \%$ de MAG avec $2,1 \%$ de MAS en 2016 ; et $11,0 \%$ de MAG avec 2,4\% de MAS en 2017 (7). La région de Koulikoro a enregistré une prévalence de $12,5 \%$ de MAG avec $2,3 \%$ de MAS en 2015, une prévalence de $10,6 \%$ de MAG avec 1,5\% de MAS en 2016 et une prévalence de $7,8 \%$ de MAG avec 1,3\% de MAS en 2017 (7). Cela dénote d'une situation stagnante et préoccupante de malnutrition des moins de 5 ans sur le plan national. Au regard de l'échelle de classification de I'OMS, la prévalence de malnutrition aigüe globale demeure élevée, car comprise entre 10 et $14 \%$ (8). Une situation nutritionnelle stagnante et aggravante des enfants de moins de 5ans nécessite des actions appropriées, adaptées et durables nécessitant des réponses de prise en charge efficace de millions d'enfants souffrant de malnutrition aigüe modérée pour les sauver de la malnutrition aigüe sévère et de la mort, à travers l'implication prioritaire des communautés et des ressources locales (9).

L'approche à base communautaire consiste à détecter à temps les cas de malnutrition aiguë dans la communauté et à fournir, lorsqu'il n'y a pas de complications médicales, un régime thérapeutique adéquat en utilisant des aliments nutritifs disponibles localement et en y ajoutant des suppléments de micronutriments (2). Le but de cette étude était de contribuer à la valorisation des produits locaux dans la lutte contre la malnutrition aigüe modérée dans la région de Koulikoro.

\footnotetext{
Matériel et Méthodes :

Type et période d'étude : Nous avons mené une étude transversale du 1er Novembre 2017 au 31 Mars 2018.

Population d'étude : II s'agissait des enfants de 6 et 59 mois dont l'état nutritionnel n'était pas satisfaisant confirmé par les mesures anthropométriques, résidant dans les villages de Tienfala et dont les parents consentaient à participer à l'étude.

Technique d'échantillonnage et taille de l'échantillon: Un dépistage actif de masse de la malnutrition a été organisé avec l'appui technique des
}

agents du centre de santé, et un total de douze enfants malnutris aigus modérés ont été retrouvé dans l'ensemble des villages.

Nous avons procédé à une sélection raisonnée, en choisissant la commune de Tienfala et ses villages par leurs accessibilités.

Collection des données : elles ont été d'une part issues de l'Enquête Nationale sur la Sécurité Alimentaire et Nutritionnelle (ENSAN) de mars 2016, puis suite au traitement des sujets sélectionnés pour les tests expérimentaux de dégustation et d'acceptabilité.

Traitement et analyse des données: Les données d'enquête ont été analysées à l'aide des logiciels 'Nutrisurvey' pour calculer la valeur nutritionnelle des aliments et 'SPSS version 21' pour réaliser l'analyse de la situation nutritionnelle de la zone d'étude.

Produits végétaux : Les produits étaient : la poudre des feuilles de Moringa oleifera, les graines de Soja, de sorgho, de maïs et de niébé.

Transformation des produits : II s'agit de procéder de transformation de Maïs et Sorgho passant par le triage, le décorticage, le lavage, le séchage, la mouture et le tamisage ; Soja et Niébé par le triage, le décorticage, le lavage, le séchage, la torréfaction, la mouture et le tamisage ; et le Sorgho en malt par le triage, le lavage, le maltage, le séchage, la mouture et le tamisage pour obtenir la farine. Les farines obtenues étaient additionnées et mélangées les unes aux autres suivant chaque formule avant leur emballage dans les sacs plastiques.

Formules alimentaires: En fonction des disponibilités des produits alimentaires de la zone étudiée et aussi des besoins nutritionnels des enfants cibles, nous avons proposé trois formules différentes pour la préparation de notre de farine de complément (tableau1).

Pour l'élaboration des formules alimentaires, nous avons utilisé les produits locaux disponibles dans la zone d'intervention. II s'agit du maïs et du sorgho comme sources principales d'énergie, le niébé et le soja comme sources principales de protéine et le Moringa comme source en vitamines et sels minéraux. Ces ingrédients ont été pris dans des proportions différentes pour la conception de chaque formule en tenant compte de la valeur énergétique que doit apporter la farine pour couvrir le besoin journalier d'un enfant malnutri (1000 $1500 \mathrm{Kcal} / \mathrm{jr}$ soit $10-15 \%$ de protéines, $30-35 \%$ de lipides et $55 \%$ de glucides), les proportions de nutriments présentées doivent être suffisantes pour satisfaire le besoin de chaque enfant malnutri (10). Les différentes valeurs obtenues pour la composition nutritionnelle lors de cette opération ont conduit aux 3 résultats pour les 3 formules ci-dessous :

- La formule alimentaire 1 à une valeur énergétique de 1055,7 kcals provenant de $19 \%$ de protéines, $50 \%$ de glucides et $30 \%$ de lipides, avec des vitamines hydrosolubles (A, B1, B2, C); dont la valeur analysée des vitamines $B 1$ et $B 2$ sont élevées dépassant parfois celle recommandée et des minéraux tels que: sodium, 
potassium, calcium, magnésium, phosphore, fer, zinc dont la valeur analysée, du fer est élevé dépassant parfois celle recommandée.

- La formule alimentaire 2 à une valeur énergétique de 1063,9 kcals avec $16 \%$ de protéine, $54 \%$ de glucides et $30 \%$ de lipides; avec des vitamines (A, B1, B2, B6 et C) ; dont la valeur analysée des vitamines B1 et B2 sont élevées dépassant parfois celle recommandée, et des minéraux tels que: sodium, potassium, calcium, magnésium, phosphore, fer, zinc dont la valeur analysée, du fer est élevé dépassant parfois celle recommandée.

- La formule alimentaire à une valeur énergétique de $1013,1 \mathrm{kcals}$ avec $15 \%$ de protéine, $54 \%$ de glucides et $31 \%$ de lipides; avec des vitamines (A, B1, B2, B6 et C); dont la valeur analysée des vitamines $B 1, B 2$ et $B 6$ sont élevées dépassant parfois celle recommandée, et des minéraux tels que: sodium, potassium, calcium, magnésium, phosphore, fer, zinc dont la valeur analysée, du magnésium, phosphore et fer sont élevé dépassant parfois celle recommandée.

Préparations : La préparation était la même pour les trois différentes formules. Les ingrédients étaient : $225 \mathrm{gr}$ de farine mélangée de céréales en sachets, $25 \mathrm{ml}$ d'huile, $45 \mathrm{gr}$ le sucre et l'eau pour la formule $1 ; 225 \mathrm{gr}$ de farine mélangée de céréales en sachets, $25 \mathrm{ml}$ d'huile, $40 \mathrm{gr} \mathrm{le}$ sucre et l'eau pour la formule $2,220 \mathrm{gr}$ de farine mélangée de céréales en sachets, $25 \mathrm{ml}$ d'huile, $20 \mathrm{gr}$ le sucre et l'eau pour la formule 3 (la quantité d'eau à utiliser était de quatre volumes d'eau pour un volume de sachets de farine). le contenu du sachet a été versé dans une tasse puis un tiers de la quantité totale d'eau y a été ajouté, le tout a été mélangé jusqu'à l'obtention d'une solution homogène. Le reste de l'eau a été mis dans la marmite et laissé bouillir au feu. A l'ébullition, la solution de farine préalablement mélangée a été ajoutée au contenu de la marmite puis mélanger quelques secondes et laisser cuire 15 minutes à feu doux. Après la cuisson, I'huile et le sucre y a été ajoutés puis mélangés jusqu'à obtention d'un mélange homogène avant de le laisser refroidir avant la consommation.

\section{Résultats :}

\section{La malnutrition dans la région de Koulikoro :}

Il ressort des données de mesure du périmètre brachial, que la situation nutritionnelle se caractérise par une malnutrition aigüe globale de 1,3\%, montrant les cercles les plus touchés que sont Dioïla, Kolokani, Banamba, et Nara avec respectivement des prévalences moyennes de 2,$2 ; 1,9 ; 1,8$ et $1,1 \%$ de malnutrition aigüe globale (fig1). La prévalence du risque de malnutrition aigüe (RMA) était de $3,3 \%$ dans la région de Koulikoro et celle de la forme modérée était de 1,2\%.

Établissement et test des formules alimentaires :

La conception des trois formules de bouillies ont êtes réalisé en tenant compte de la valeur énergétique que doit apporter la farine pour couvrir le besoin journalier d'un enfant malnutri (1000 - $1500 \mathrm{Kcal} / \mathrm{jr}$ soit $10-15 \%$ de protéines, $30-35 \%$ de lipides et $55 \%$ de glucides) (Tableau II).
Le test de dégustation des 3 formules de bouillie par les femmes et leurs enfants ont permis de constater que dans l'ensemble, la première formule était bien apprécie par les enfants (tableau III).

\section{Discussions}

Les résultats ci-dessus portent essentiellement sur l'enquête nutritionnelle de l'ENSAN MALI (Enquête Nationale sur la Sécurité Alimentaire et Nutritionnelle), Mars 2016 dans la région de Koulikoro. Afin de développer des formules alimentaires basées sur les produits locaux et tester ces formulations comme farines de suppléments et de compléments, il est nécessaire de comprendre les causes de la malnutrition dans la zone cible.

II ressort des données de mesure du PB, que les enfants de 6-59 mois sont affectés par une prévalence de malnutrition aigüe globale de 1,3\% montrant les cercles les plus touchés qu'étaient Dioïla, Kolokani, Banamba, et Nara avec respectivement des prévalences moyennes de 2,$2 ; 1,9 ; 1,8$ et $1,1 \%$; il apparait que d'une part, la situation reflète des tendances similaires comparées aux données SMART 2016 qui était de 1,6\% dans l'ensemble de la région de Koulikoro (1); et d'autre part cette prévalence est relativement plus basse comparée à la dernière enquête ENSAN de février 2017 qui était de $3,5 \%$ de malnutrition aigüe globale, d'où une tendance à la baisse (11)

La situation pour les formes de malnutrition offre une autre physionomie. La prévalence du risque de malnutrition aigüe (RMA) est plus élevée dans la région de Koulikoro que les autres formes de malnutritions suivi par la forme modérée de la malnutrition avec une prévalence respective de $3,3 \%$ de RMA et $1,2 \%$ de MAM, dont les cercles qui avaient un taux relativement élevé de risque de malnutrition comparativement aux autres étaient Kangaba, Koulikoro, Kolokani et Dioilla (avec respectivement 7,$8 ; 3,1 ; 3,0$ et $2,9 \%$ ) et par la malnutrition modérée sont Kolokani, Banamba, Dioïla et Nara (avec respectivement 1,$9 ; 1,8 ; 1,7$ et $1,1 \%$ ). La forme sévère ne représente que $0,1 \%$ dans l'ensemble de la région de Koulikoro avec le seul cercle de Dioïla affecté avec $0,5 \%$ de MAS ; comparées aux données SMART 2016, la situation reflète des tendances similaires avec une prévalence de malnutrition modérée $(1,0 \%)$ plus élevée que la forme sévère $(0,6 \%)(6)$, et cette prévalence est basse comparativement à la dernière enquête ENSAN du février 2017 qui était de $0,1 \%$ de forme sévère de malnutrition aiguë (11).

Les dégustateurs devaient déterminer les propriétés organoleptiques de chaque formule de bouillie. II s'agissait donc pour cela d'apprécier le goût, la couleur, l'acidité et l'odeur des deux bouillies pour chaque formule de farine. Ainsi :

Par rapport au goût, les bouillies de la formule 1 et la formule 2 ont été peu appréciées, car la quantité de 
sucre était insuffisante. Par contre, la quantité de sucre pour la formule 3 était très satisfaisante.

L'odeur du niébé se faisait sentir dans la formule 1, mais les deux autres formules n'avaient pas d'odeur particulièrement gênante.

L'acidité et la couleur verdâtre des différentes formulations étaient très bien appréciées.

Au final, la formule trois a été la formule la plus appréciée par les accompagnantes des enfants.

Cette phase de dégustation par les enfants a été réalisée au CSCom de Tienfala. Cela a été un moment très agréable, car les enfants ont apprécié les bouillies de chaque formule.

Sur l'ensemble des enfants ayant participé, seuls deux (encore en phase d'allaitement de moins de 6 mois) n'ont pas consommé de ration. Et toutes les bouillies ont été acceptées et appréciées. Tous les enfants ont complètement consommé leurs rations. Ainsi nous pouvons penser que les trois différentes formules proposées ont été acceptées par tous les enfants.

Les formules ainsi dégustées peuvent donc toutes les trois être utilisées tout comme des farines de complément ou de supplément respectivement pour la prévention et le traitement des cas de malnutrition aigüe modérée. II faut noter cependant qu'en égard à l'enthousiasme affichée des enfants pour les bouillies, le schéma de distribution des bouillies a permis de montrer que la première formule était la plus appréciée par les enfants.

\section{Conclusion :}

La présente étude a consisté à déterminer l'état nutritionnel des enfants de 6-59 mois et proposer des formules d'aliments de haute valeur nutritionnelle à base des produits locaux (sorgho, maïs, niébé et soja) qui pourraient aussi véhiculer des micronutriments pour améliorer l'état nutritionnel des enfants dans la zone cible. Les trois différentes formules de bouillie lors de la dégustation ont été acceptées et appréciées par les enfants. Ainsi, compte tenu de leur haute valeur nutritionnelle, elles pourraient être utilisées comme farines de complément/ supplément pour les cas de la malnutrition aigüe modérée.

II apparait important de valoriser les produits locaux pour la conception des farines de complément ou de supplément, d'appuyer les communautés dans la formulation de farine de complémentation basée sur les produits locaux pour une meilleure couverture des enfants de moins de 5 ans dans la zone d'étude ; de former les femmes aux techniques améliorées de transformation et de conservation des produits locaux, et de former les femmes des associations sur la conception des formules alimentaires.

\section{Références :}

1. USAID. Community-Based Management of Acute Malnutrition: Technical Guidance Brief [Internet]. USAID; 2019 [cité 22 déc 2019]. Disponible sur: https://www.usaid.gov/global-health/healthareas/nutrition/technical-areas/community-basedmanagement-acute-malnutrition

2. Organisation mondiale de la Santé, Programme alimentaire mondial, Comité permanent de la nutrition du Système des Nations Unies, Fonds des Nations Unies pour l'enfance. Prise en charge communautaire de la malnutrition aiguë sévère [Internet]. OMS Genève; 2007 [cité 21 déc 2019]. Disponible sur:

http://www.who.int/nutrition/publications/severemalnutritio n/9789280641479/fr/

3. Évaluation Office, UNICEF. ÉVALUATION DE

LA PRISE EN CHARGE COMMUNAUTAIRE DE LA MALNUTRITION AIGUË (PCMA) : ÉTUDE DE CAS DU

TCHAD [Internet]. UNICEF; 2012 [cité 22 déc 2019].

Disponible sur:

https://www.unicef.org/evaldatabase/files/Evaluation_Pris e_en_charge_communautaire_de_la_malnutrition_aigue _Tchad_2012.pdf

4. Choudhury N, Ahmed T, Hossain Mdl, Mandal BN, Mothabbir G, Rahman M, et al. Community-Based Management of Acute Malnutrition in Bangladesh:

Feasibility and Constraints. Food Nutr Bull. 1 juin 2014;35(2):277-85.

5. Maleta K, Amadi B. Community-Based Management of Acute Malnutrition (CMAM) in SubSaharan Africa: Case Studies from Ghana, Malawi, and Zambia. Food Nutr Bull. 1 juin 2014;35(2_suppl1):S34-8. 6 CPS/SSDSPF, INSTAT, INFO-STAT. Enquête Démographique et de Santé au Mali 2012-2013 [Internet]. ICF International, Rockville, Maryland, USA; 2014 [cité 24 déc 2017]. Disponible sur: http://www.sante.gov.ml/index.php/annuaires/send/8enquetes-demographiques-de-sante/4-eds-v-2013 7. Institut National de la Statistique (INSTAT). Enquête Nationale Nutritionnelle Anthropométrique et de Mortalité rétrospective. 2017;131.

8. OMS, UNICEF. Normes de croissance OMS et identification de la malnutrition aiguë sévère chez l'enfant [Internet]. OMS; 2009 [cité 22 déc 2019]. Disponible sur: https://www.who.int/nutrition/publications/severemalnutriti on/9789242598162_fre.pdf

9. Ag lknane A. Actions Essentielles en Nutrition. Cours de Nutrition présenté à: Formation en Santé Publique; 2017; DER de Santé Publique.

10. Ministère de la Santé. Protocole de Prise en Charge Intégrée de la Malnutrition Aiguë au Mali. Version révisée en 2011 [Internet]. Direction Nationale de la Santé, Division Nutrition; 2011. Disponible sur: https://www.humanitarianresponse.info/sites/www.human 
itarianresponse.info/files/documents/files/Protocole_PECI MA_Mali\%2023_06-2012VF.pdf

11. SAP, PAM, FAO, FEWS NET, CLUSER

SECURITE ALIMENTAIRE. Rapport de Synthese -

Enquête Nationale sur la Sécurite Alimentaire et

Nutritionnelle, Février 2017 (ENSAN Mali) [Internet]. Mali: Système d'Alerte Précoce (SAP); 61 [cité 22 déc 2019].

Report No.: Version provisoire. Disponible sur:

https://www.humanitarianresponse.info/en/operations/mal i/document/enquete-nationale-sur-la-securite-alimentaireet-nutritionnelle-f\% $\%$ C3\%A9vier

Liste des tableaux et figures :

Tableau I : Différentes formulations de la farine de complément

\begin{tabular}{lccc}
\hline Produits & Formule & Formule & \multicolumn{2}{l}{ Formule } \\
& $\mathbf{1}$ & $\mathbf{2}$ & $\mathbf{3}$ \\
\hline Maïs & - & - & 120 \\
Niébé & 100 & 65 & - \\
Sorgho 'Fambé' & - & 85 & - \\
Sorgho 2 & 100 & - & - \\
Malt & - & 50 & - \\
Moringa oleifera & 25 & 25 & 35 \\
Soja & - & - & 65 \\
Huile & 25 & 25 & 25 \\
Sucre & 45 & 40 & 20
\end{tabular}

\begin{tabular}{lrrr} 
V.E (Kcal) & 1055,7 & 1063,9 & 1013,1 \\
\hline
\end{tabular}

Tableau III : Appréciation de la bouillie par les accompagnantes au CSCom de Tienfala le 14 mars 2018

\begin{tabular}{|c|c|c|c|c|}
\hline Dégustateur & Goût & Odeur & Couleur & Acidité \\
\hline \multicolumn{5}{|c|}{ Formule 1} \\
\hline 1 & ++ & + & +++ & +++ \\
\hline 2 & ++ & + & +++ & +++ \\
\hline 3 & ++ & ++ & +++ & +++ \\
\hline 4 & ++ & + & +++ & +++ \\
\hline \multicolumn{5}{|c|}{ Formule 2} \\
\hline 1 & ++ & +++ & +++ & +++ \\
\hline 2 & ++ & +++ & +++ & +++ \\
\hline 3 & ++ & +++ & +++ & +++ \\
\hline 4 & ++ & +++ & +++ & +++ \\
\hline \multicolumn{5}{|c|}{ Formule 3} \\
\hline 1 & +++ & +++ & +++ & +++ \\
\hline 2 & +++ & +++ & +++ & +++ \\
\hline 3 & +++ & +++ & +++ & +++ \\
\hline 4 & +++ & +++ & +++ & +++ \\
\hline
\end{tabular}

Légende : + : moins appréciée ; ++ : bien appréciée ; +++ : très bien appréciée 
Tableau I : La valeur énergétique et valeur en macronutriment

\begin{tabular}{ccccc}
\hline Formule alimentaire & \multicolumn{3}{c}{ Nutriments } \\
\hline & Énergie & Protéines & Lipides & Glucides \\
\cline { 2 - 5 } Formule alimentaire 1 & $1055,7 \mathrm{kcal}$ & $39,3 \mathrm{~g}(19 \%)$ & $28,6 \mathrm{~g}(30 \%)$ & $102,7 \mathrm{~g}(50 \%)$ \\
Formule alimentaire 2 & $1063,9 \mathrm{kcal}$ & $35,5 \mathrm{~g}(16 \%)$ & $29,4 \mathrm{~g}(30 \%)$ & $117,3 \mathrm{~g}(54 \%)$ \\
Formule alimentaire 3 & $1013,1 \mathrm{kcal}$ & $41,2 \mathrm{~g}(15 \%)$ & $38,6 \mathrm{~g}(31 \%)$ & $144,6 \mathrm{~g}(54 \%)$ \\
\hline
\end{tabular}

Fig.1 : prévalence de la malnutrition aigüe globale par cercle de la région de Koulikoro (ENSAN MALI Mars 2016)

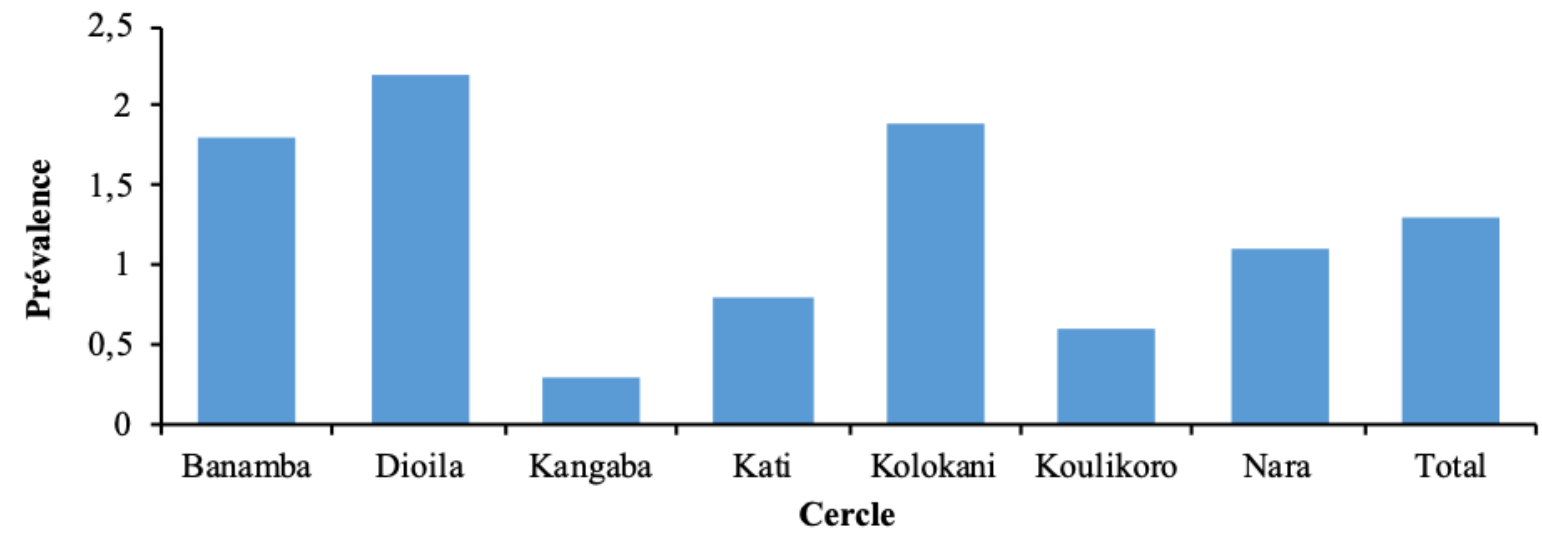

Fig.2 : prévalence des formes de malnutrition aigüe par cercle de la région de Koulikoro (ENSAN MALI Mars 2016).

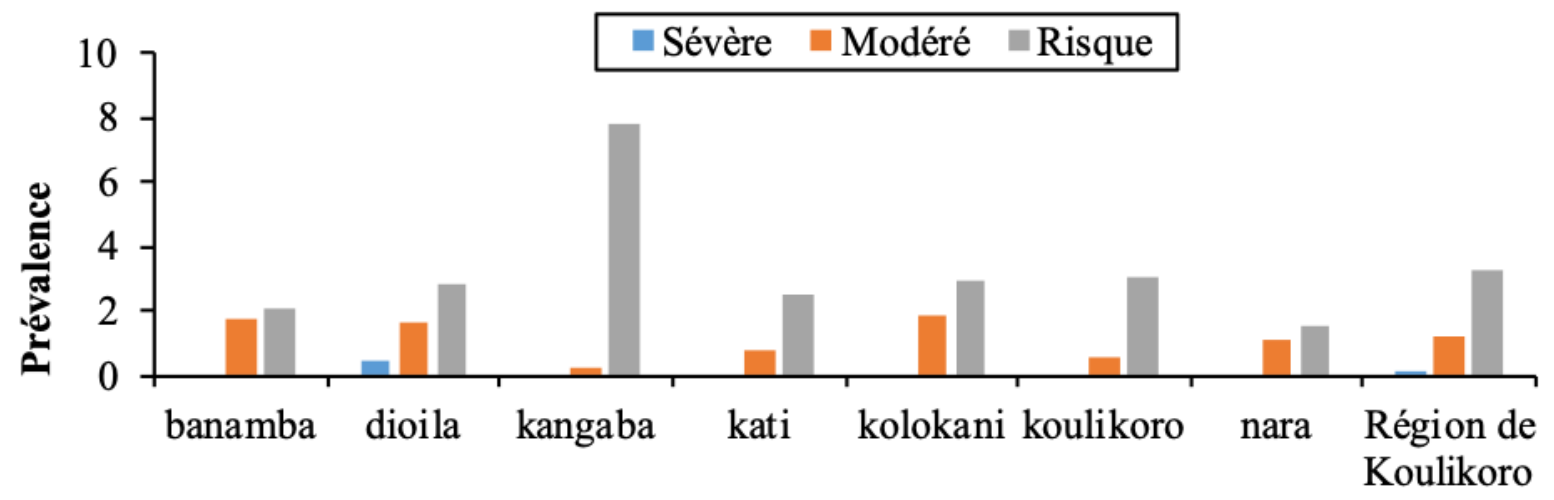

Localité 